\title{
An unbalanced translocation unmasks a recessive mutation in the follicle-stimulating hormone receptor (FSHR) gene and causes FSH resistance
}

\author{
Amla Kuechler ${ }^{\star, 1}$, Berthold P Hauffa ${ }^{2}$, Angela Köninger ${ }^{3}$, Gunnar Kleinau ${ }^{4}$, Beate Albrecht ${ }^{1}$, \\ Bernhard Horsthemke ${ }^{1}$ and Jörg Gromoll ${ }^{5}$
}

Follicle-stimulating hormone (FSH) mediated by its receptor (FSHR) is pivotal for normal gametogenesis. Inactivating FSHR mutations are known to cause hypergonadotropic hypogonadism with disturbed follicular maturation in females. So far, only very few recessive point mutations have been described. We report on a 17-year-old female with primary amenorrhea, hypergonadotropic hypogonadism and disturbed folliculogenesis. Chromosome analysis detected a seemingly balanced translocation $46, \mathrm{XX}, \mathrm{t}(2 ; 8)(\mathrm{p} 16.30 \mathrm{r} 21 ; \mathrm{p} 23.1)$ mat. FSHR sequence analysis revealed a novel non-synonymous point mutation in exon 10 (c.1760C $>$ A, p.Pro587His), but no wild-type allele. The mutation was also found in the father, but not in the mother. Furthermore, molecular-cytogenetic analyses of the breakpoint region on chromosome 2 showed the translocation to be unbalanced, containing a deletion with one breakpoint within the FSHR gene. The deletion size was narrowed down by array analysis to approximately $163 \mathrm{~kb}$, involving exons 9 and 10 of the $F S H R$ gene. Functional studies of the mutation revealed the complete lack of signal transduction presumably caused by a changed conformational structure of transmembrane helix 6 . To our knowledge, this is the first description of a compound heterozygosity of an inactivating FSHR point mutation unmasked by a partial deletion. This coincidence of two rare changes caused clinical signs consistent with FSH resistance.

European Journal of Human Genetics (2010) 18, 656-661; doi:10.1038/ejhg.2009.244; published online 20 January 2010

Keywords: FSH receptor deficiency; FSHR mutation; chromosome 2p16.3; translocation; hypergonadotropic hypogonadism

\section{INTRODUCTION}

Follicle-stimulating hormone (FSH) and its corresponding receptor (FSHR) are essential factors for regular gonadal development, sexual maturation at puberty and gamete production during the fertile period in both sexes. Normal FSH function regulates maturation of follicles and estrogen production in females and supports spermatogenesis in males. ${ }^{1,2}$ The FSHR (OMIM $\left.{ }^{*} 136435\right)$, a G-protein-coupled receptor, is expressed by granulosa cells in the ovary and Sertoli cells in the testis. The FSHR encoding gene was mapped to the short arm of chromosome 2 in 2p16.3. ${ }^{3}$ It consists of 10 exons and spans about $54 \mathrm{~kb}$ of genomic DNA. ${ }^{4}$ Mutations can be activating or inactivating. In females, inactivating mutations result clinically - depending on the degree of inactivation - in primary amenorrhea, secondary amenorrhea or premature ovarian failure with the histopathological finding of impaired follicular maturation (ovarian dysgenesis 1, OMIM \#. 233300). Total FSHR inactivation is characterized by infertility with an early block in maturation and high numbers of primordial follicles as in prepubertal stage and hypoplastic ovaries. The first FSHR mutation, a homozygous missense mutation (566C $>\mathrm{T}$, Ala189Val), was described in 1995 by Aittomäki et al in several Finnish patients with primary amenorrhea, hypergonadotropic hypogonadism and blocked follicle maturation. ${ }^{5,6}$ Since then, only 10 more FSHRinactivating mutations have been published. ${ }^{7-13}$ The only activating mutation leading to a ligand-independent constitutive activation of the FSH receptor was identified in a hypophysectomized patient who had normal fertility under testosterone substitution alone without detectable serum gonadotropin levels. ${ }^{14}$

In addition, FSHR mutations causing ovarian hyperstimulation syndrome have recently been identified. ${ }^{15-18}$ These mutations result in a decreased ligand specificity allowing not only FSH but also hCG/TSH to stimulate the receptor. In gain-of-function mutations, the phenotypic changes are caused by heterozygosity and dominant inheritance, whereas loss-of-function mutations are mostly transmitted recessively and need homozygosity or compound heterozygosity to cause the clinical phenotype. ${ }^{19}$

In this study, we describe for the first time the compound heterozygous combination of a novel inactivating point mutation that was unmasked by a small partial deletion of the FSHR locus resulting clinically in a hypergonadotropic hypogonadism with primary amenorrhea.

\section{MATERIALS AND METHODS}

Conventional chromosome analysis

After informed consent, peripheral blood was taken from the proposita and her parents and relatives. Lymphocytes were cultured with phytohemagglutinin and

${ }^{1}$ Institut für Humangenetik, Universitätsklinikum, Universität Duisburg-Essen, Essen, Germany; ${ }^{2}$ Department of Paediatric Endocrinology and Diabetology, University Children's Hospital, University Duisburg-Essen, Essen, Germany; ${ }^{3}$ Department of Obstetrics and Gynaecology, University Hospital, University Duisburg-Essen, Essen, Germany; ${ }^{4}$ Leibniz-Institut für Molekulare Pharmakologie, Berlin, Germany; ${ }^{5}$ Center for Reproductive Medicine and Andrology, University of Münster, Münster, Germany *Correspondence: Dr A Kuechler, Institut für Humangenetik, Universitätsklinikum Essen, Hufelandstrasse 55, Essen D-45122, Germany. Tel: +49 201723 4565; Fax: +49 201723 5900; E-mail: alma.kuechler@uni-due.de

Received 7 October 2009; revised 26 November 2009; accepted 16 December 2009; published online 20 January 2010 
cells were harvested according to cytogenetic standard procedures of hypotonic treatment and methanol/acetic acid fixation (3:1). Chromosome analysis was performed using GTG-banding at the 500-550 band level (as determined by the ISCN 2009 standards).

\section{Fluorescence in situ hybridization (FISH)}

FISH was performed with whole chromosome painting probes (wcp) for chromosomes 2 and 8 (MetaSystems, Altlussheim, Germany), centromeric probes for chromosomes 8 and X (CEP8, CEPX), and LSI SRY (obtained from Abbott Molecular Inc., Downers Grove, IL, USA) and two BAC clones, both localized in 2p16.3 (RP11-19A8 and RP11-436L21, obtained from BlueGnome, Cambridge, UK). Hybridization and post-hybridization washes were carried out according to the manufacturer's protocols. Chromosomes were counterstained with DAPI (4,6-diamino-2-phenylindole). Slides were analyzed with an epifluorescent microscope Axioplan (Zeiss, Oberkochen, Germany) and images were captured using Isis software (MetaSystems, Altlussheim, Germany). FISH signals were examined both on metaphase chromosomes and interphase nuclei.

\section{DNA isolation and sequencing}

DNA was extracted from venous blood using the FlexiGene DNA Kit (Qiagen, Hilden, Germany) following the manufacturer's instructions. Each of the 10 exons of the FSHR gene (RefSeq NM_000145) was amplified from genomic DNA by PCR and sequenced as described earlier. ${ }^{20}$

\section{Microarray analysis}

SNP array analysis was carried out using the GeneChipHuman Mapping $250 \mathrm{~K}$ Sty Array according to the protocol of the manufacturer (Affymetrix, Santa Clara, CA, USA). Imaging of the microarrays was performed using the GCS3000-G7 scanner from Affymetrix. Genotype calls and probe intensity data were extracted with the GTYPE4.1 software (Affymetrix). Copy number analysis was performed using the CNAG v2.0 software (Copy Number Analyzer for GeneChip; ${ }^{21}$ http://www.genome.umin.jp). The design of the $250 \mathrm{~K}$ Array and the interpretation of data were based on the March 2006 human genome sequence assembly (UCSC hg18). Conspicuous regions were compared with known CNVs, as provided by the Database of Genomic Variants (http://projects.tcag.ca/variation/).

\section{Functional characterization of the mutation}

The identified nucleotide point mutation changing the codon for proline (CCC) into histidine (CAC) was introduced into the wt-FSHR cloned into the pSG5 plasmid by in vitro mutagenesis as previously described ${ }^{4}$ using the quickchange-sited directed mutagenesis kit (Agilent Technologies). Functional studies were performed by transient transfection of COS-7 cells (50 000 cells per well) by lipofection (Lipofectamine, GE Healthcare) using $2 \mu \mathrm{g}$ plasmid DNA/well. Twenty-four hours after transfection, cells were stimulated for $3 \mathrm{~h}$ with increasing doses of human recFSH (Gonal F, Merck-Serono). cAMP was determined by an in-house assay. cAMP values were determined in duplicates and three independent transfections were performed.

\section{Molecular homology modeling}

We generated homology models of the seven transmembrane helices, intracellular helix eight, and the helix-connecting loops for both wild-type FSHR and an inactive FSHR variant (P587H in TMH6). The models were based on rhodopsin ( $p d b$ code 2I35). FSHR-specific modifications relative to rhodopsin were made in the homology models. ${ }^{22}$ In rhodopsin, interactions between the side chains of two consecutive threonines and the helix backbone of the preceding residues cause a helical bulge in TMH2. In the FSHR, these consecutive threonines do not exist in TMH2, which suggests the presence of a regular $\alpha$-helix. At TMH5, a minor change in orientation of the N-terminal half of TMH5 (a twist of 10-15 degrees) was generated because of the lack of a proline compared with rhodopsin. Gaps of missing residues in the loops of the template structure were closed by the 'Loop Search' tool implemented in Sybyl 7.3.5 (Tripos Inc., St. Louis, MO, 63144,
USA). The generated homology models were initially subjected to conjugate gradient minimization (until converging at a termination gradient of $0.05 \mathrm{kcal} /$ $\left(\operatorname{mol}^{*} \AA\right)$ ) and secondly to molecular dynamics (MD) simulation. The MD simulation of two nanoseconds (ns) was performed in two steps: (i) $1 \mathrm{~ns}$ with fixed backbone atoms and (ii) $1 \mathrm{~ns}$ with released backbone atoms. Images were produced using PyMOL software (DeLano WL, version 099, San Carlos, CA, USA).

\section{RESULTS}

\section{Clinical report}

A 17-year-old female was referred because of a primary amenorrhea and incomplete pubertal development. Breast and pubic development corresponded to Tanner stage 3 (B3, PH3). In addition, she had lichen sclerosus et atrophicus of the vulva. Her body measurements have always been normal (at birth: $49 \mathrm{~cm}$ length ( -1.2 SDS), $3200 \mathrm{~g}$ weight ( $-0.6 \mathrm{SDS})$; at present: $159 \mathrm{~cm}$ height $(-1.56 \mathrm{SDS}), 48 \mathrm{~kg}$ weight $(-0.83 \mathrm{SDS}), 55.6 \mathrm{~cm}$ head circumference (+0.6 SDS) (according to $\left.{ }^{23-25}\right)$ ). She had a normal childhood development and did not show any dysmorphic signs. An X-ray of the left hand at age 17 years showed bone age retardation by 3-4 years. Endocrinological investigations revealed hypergonadotropic hypogonadism with prepubertal estradiol levels (results of two consecutive hormone measurements: FSH 105.9 and 121.8 IU/l (normal range, follicular phase 2.5-10.2 IU/l), LH 47.8 and $46.7 \mathrm{U} / 1$ (normal range, follicular phase 1.9-12.5 IU/l), estradiol 13.3 and $10.7 \mathrm{pg} / \mathrm{ml}$ (normal range, follicular phase $11-165 \mathrm{pg} / \mathrm{ml})$. At diagnostic laparoscopy, ovaries of normal size and a rather small uterus were seen. The histological examination of ovarian tissue showed a disturbed folliculogenesis with a high number of primordial follicles, but complete absence of secondary or tertiary follicles. The lack of oocyte maturation combined with hypergonadotropic hypogonadism led us to hypothesize a defective FSH action as the cause of the primary amenorrhea.

\section{Cytogenetic and molecular data}

Chromosome analysis. Chromosome analysis was performed as the first step to identify the cause of the primary amenorrhea. This showed a female karyotype with two structurally normal $\mathrm{X}$-chromosomes but a translocation between one chromosome 2 and one chromosome 8 [46,XX,t $(2 ; 8)(\mathrm{p} 16.3$ or 21;p23.1)]. Wcp probes for chromosomes 2 and 8 confirmed the translocation partners and excluded a more complex involvement of other chromosomes (see Figure 1a). The translocation appeared to be cytogenetically balanced. This was seemingly confirmed by the finding of the same translocation in the unaffected mother (normal male karyotype in the father). The translocation was absent in the maternal brother and sister as well as in the patient's brother. Maternal grandparents were not available for chromosome analysis. As this translocation initially did not explain the primary amenorrhea, FISH with CEPX and SRY was performed and a numerical gonosomal mosaicism or an involvement of SRY was excluded in 100 cells (analysis of 40 metaphase spreads and 60 interphase nuclei, data not shown).

FSHR mutation analysis. Owing to the clinical diagnosis of FSH resistance, we performed sequence analysis of the FSHR gene. This revealed a single non-synonymous seemingly homozygous point mutation in exon 10 (CCC to CAC, Pro587His) but no wild-type allele (see Figure 2a).

Screening the family members of the proposita revealed that the Pro587His FSHR mutation was transmitted by the father 
(heterozygous carrier), whereas the mother was not affected. The patient's brother was also found to be heterozygous for this mutation.

Breakpoint characterization on chromosome 2p16.3. As the FSHR gene was mapped to the same chromosomal region as the cytogenetically defined translocation breakpoint (2p16.3or21), ${ }^{3}$ the next step was to find out whether the breakpoint affected the FSHR gene. We hybridized two BAC probes, RP11-436L21 containing exon 1, and RP11-19A8 containing exons 5-10 of the FSHR gene (for exact localization of $\mathrm{BAC}$ probes in relation to the FSHR gene, see
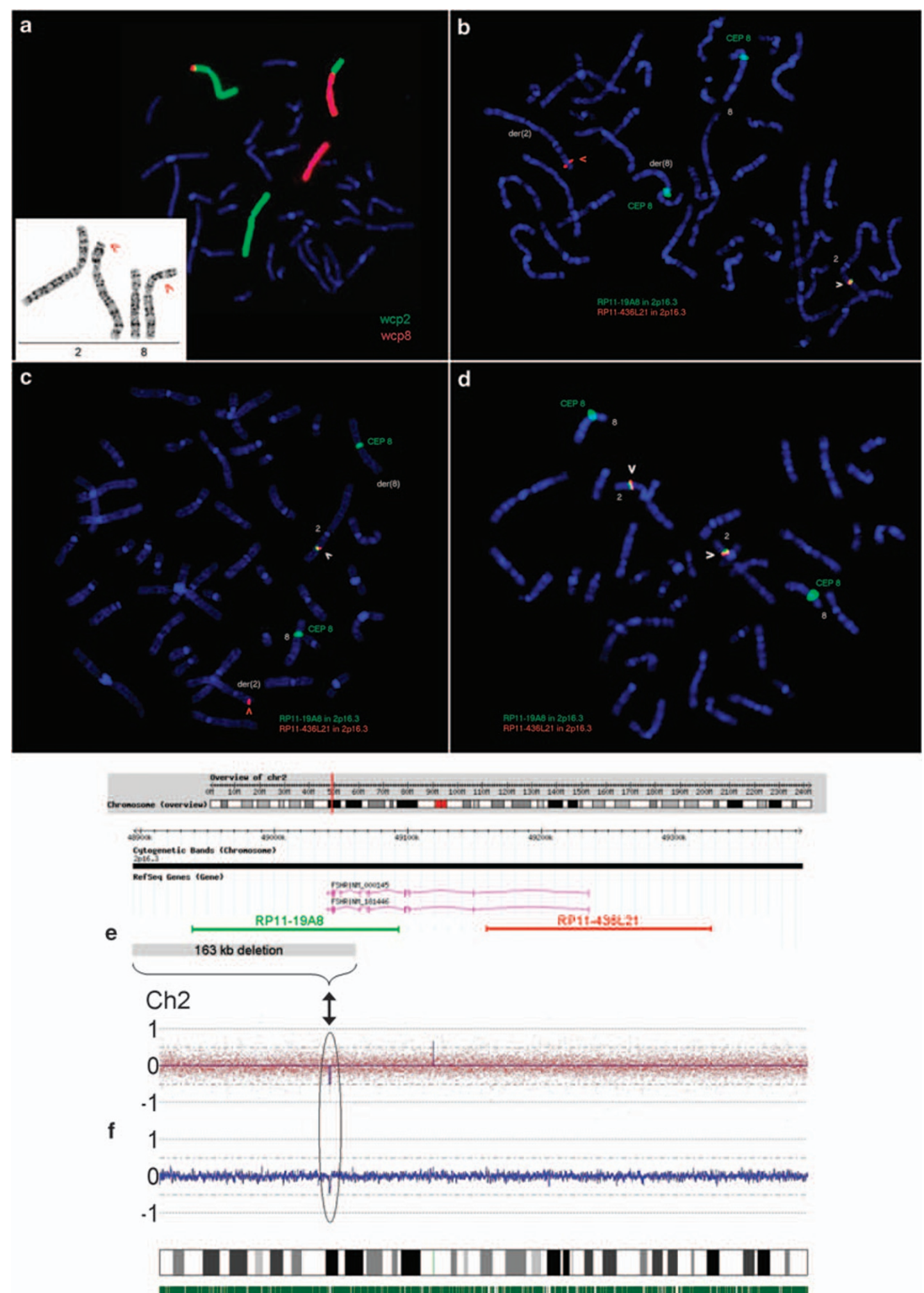
Figure 1e). This showed two normal signals for RP11-436L21 on both the normal chromosome 2 and the derivative chromosome der(2), whereas clone RP11-19A8 only hybridized to the normal chromosome 2. The second signal was absent on the $\operatorname{der}(2)$ as well as on the $\operatorname{der}(8)$. This showed the translocation $\mathrm{t}(2 ; 8)$ to be associated with a partial deletion of the FSHR gene (see Figure 1b) with one deletion breakpoint localized between the two BAC clones within the gene.

Hybridization of the two BAC clones to chromosomes of the proband's mother (also translocation carrier) revealed the same result of a deleted RP11-19A8 signal on the der(2) (see Figure 1c). Hybridization to chromosomes of the father (Figure 1d), the brother and the maternal aunt and uncle (all carry normal karyotypes) confirmed the expected normal signal constellation for both BACs on chromosome 2 .

Microarray analysis showed a heterozygous deletion of about $162.7 \mathrm{~kb}$ in size in $2 \mathrm{p} 16.3$ containing approximately 30 SNPs. The proximal breakpoint was located between the flanking SNP rs6729545 (position chr.2: 48887 046) and the first deleted SNP rs9636432 (chr.2: $48895903 \mathrm{bp}$ ), the distal breakpoint between the last deleted SNP rs2268359 (chr.2: $49058614 \mathrm{bp}$ ) and the flanking SNP rs13396575 (chr.2: 49065 417; UCSC hg18). The deleted region included exons 9 and 10 of the FSHR gene (see Figure 1e and $\mathrm{f}$ ).

Functional characterization of the mutation. Functional studies on transiently transfected COS-7 cells using the wt-FSHR and the
Pro587His mutated FSHR revealed a normal increase of cAMP production on FSH stimulation (up to 8-fold increase) in the wild type, which was completely absent in the mutated FSHR (Figure $2 b$ ). The functional data therefore strongly indicate that this mutation abolishes normal signal transduction.

Structural consequences of the Pro587His FSHR mutation. Pro587 is located in the sixth transmembrane helix (TMH6) and causes a kink distortion, which is important for activation and signal transduction in class A G-protein-coupled receptors (Figure 3). On the basis of molecular dynamic simulations, substitution of Pro to His changes the structure of TMH6. As a consequence, the signaling associated interfaces between TMH5 and TMH6, or ECL2 and TMH6 are altered in spatial distance and in intramolecular side chain interactions.

\section{DISCUSSION}

We report here on a compound heterozygosity of a novel nonsynonymous Pro587His FSHR point mutation, unmasked by a partial deletion of the FSHR gene. Owing to the absence of a wild-type allele, a homozygous mutation was suspected initially, but mutation analysis in the parents failed to detect it in the mother. Therefore, a deletion of the second allele became most likely, in particular because the breakpoint of the familial translocation seemed to be in close proximity to the FSHR locus. The mechanism of unmasking a recessive
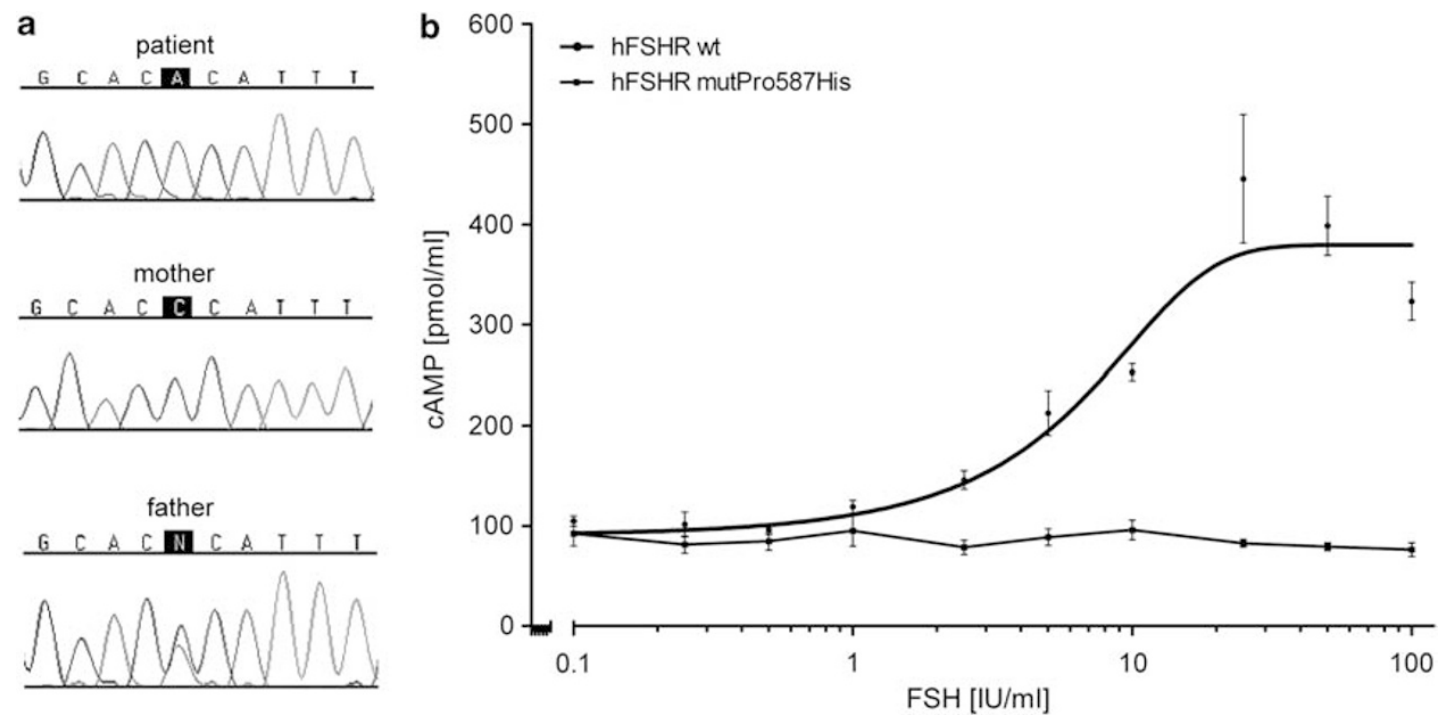

Figure 2 (a) Novel FSHR mutation Pro587His in Exon 10. Results of direct sequencing show the mutant allele (CAC) in the patient with absence of a wild-type allele, the homozygous wild-type allele in the patient's mother (CCC) and the heterozygous mutation (CNC) in the patient's father. (b) Functional characterization of the Pro587His FSHR mutation. COS-7 cells were transiently transfected with either the wild-type (hFSHwt)- or the Pro587His (hFSHRmutPro587His)- FSH receptor. After transfection, cells were stimulated with increasing doses of human rec hFSH and cAMP production was determined. cAMP values were measured in duplicates and three independent transfections were performed.

Figure 1 (a) Partial karyotype of the patient. Chromosome analysis after GTG banding revealed a seemingly balanced translocation between the short arms of one chromosome 2 and one chromosome 8 (shown on the lower left, marked by red arrows), resulting in karyotype: 46,XX,t(2;8)(p16.3or21;p23.1). The translocation partners were confirmed and a more complex involvement of further chromosomes was excluded by FISH using whole chromosome painting probes wcp2 (labeled in green) and wcp8 (labeled in red). (b-d) Breakpoint characterization on chromosome 2p16.3 by fluorescence in situ hybridization. The cytogenetically defined translocation breakpoint in 2p16.3or21 was first characterized by two BAC probes: RP11-436L21 (labeled in red) containing exon 1, and RP11-19A8 (labeled in green) containing exons 5-10 of the FSHR (for localization of BAC clones related to the FSHR gene see e). A deletion of clone RP11-19A8 on the der(2) (marked by a red arrowhead) was detected in the patient (b) and also in her mother who is also a translocation carrier (c) but not in the father (d). This showed the translocation $\mathrm{t}(2 ; 8)$ to be unbalanced and associated with a partial deletion of the FSHR gene. (f) Array analysis using GeneChipHumanMapping $250 \mathrm{~K}$ Sty Array (Affymetrix). The copy number plot (log2 ratio) of the entire chromosome 2 shows a heterozygous deletion (marked with a black arrow) of about $162.7 \mathrm{~kb}$ in size in 2p16.3, including exons 9 and 10 of the FSHR gene. 


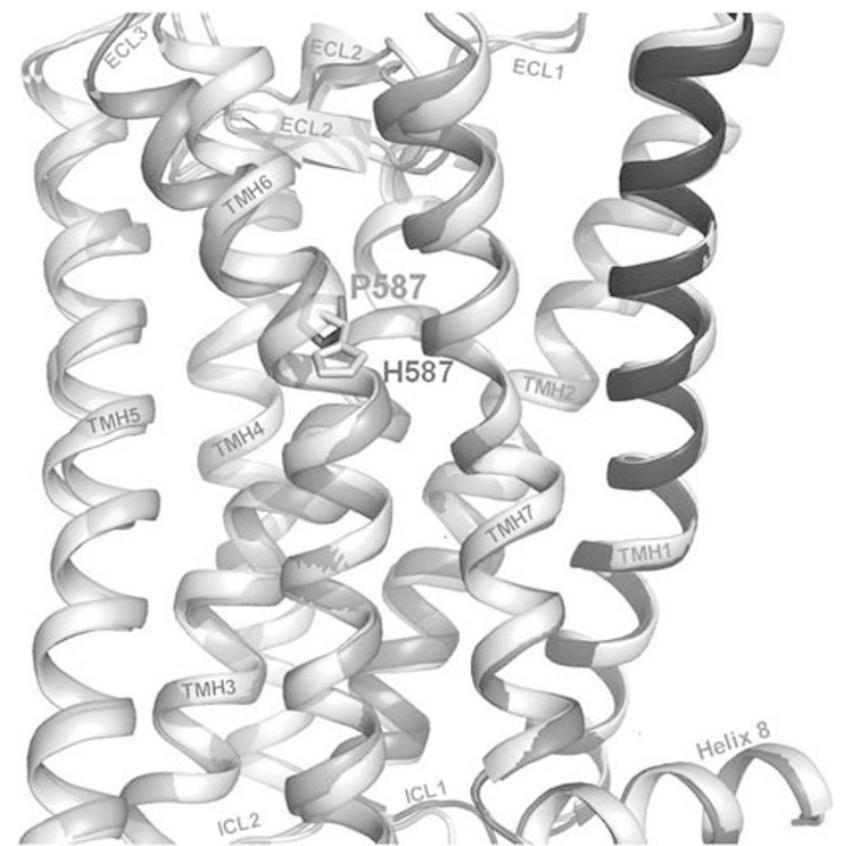

Figure 3 Molecular homology models of the wild-type and the mutated hFSHR. The backbones of wild-type (coloured rainbow) and Pro-His mutated hFSHR (white) are overlaid. The wild-type amino acid, Proline, is shown at position 587 as well as the mutated histidine side chain. The kink distortion in helix 6 (orange) is caused by the proline and intramolecular side chain interaction between the specifically arranged helical conformations (eg, TMH6 to TMH5 and TMH7). The kink is an important pivot during receptor activation. Substitution of Pro to $\mathrm{His}$ is likely to cause structural modifications in TMH6 and consequently also between structural components, eg, the important interfaces between TMH5 and TMH6 or between TMH6 and ECL2. The evoked shift therefore changes stabilizing intramolecular interactions between the helices and leads to a loss of intrinsic signaling capability because of an altered basal conformation.

mutation by a deletion of the second allele was first described in 1993 by Rinchik et $a l^{26}$ in a patient with Prader-Willi syndrome and oculocutaneous albinism. Since then, it has been reported in several conditions, but not in combination with an FSHR mutation.

We could prove our assumption by confirming the deletion by FISH and array analysis. According to the array results, the deleted BAC clone (RP11-19A8) spans the breakpoint and is deleted for the most part. It can be assumed that the remaining portion is too small to generate a detectable fluorescence signal. Searching the Database of Genomic Variants (http://projects.tcag.ca/variation/) for known copy number variations in this region, one copy number loss was found (Variation_8945, Pinto et al, 2007; one loss in 776 controls) that only in part overlapped with the deleted region in our patient.

It remains unknown whether the deletion in this family predisposed to the formation of the translocation or whether the deletion is a result of the translocation. This cannot be elucidated further as the grandparents are not available for investigation.

The deletion contains exons 9 and 10 of the FSHR gene. Exon 10 is its largest exon, constituting the entire transmembrane helix of the receptor, the intracellular domain and the C-terminal part of the extracellular domain. Exons 1-9 encode the extracellular domain. ${ }^{4}$ Therefore, a deletion of these two exons should lead to a complete loss-of-function of this allele, if the mRNA is translated at all.

Up to now, only point mutations (either homozygous or compound heterozygous) have been published in patients with variable clinical phenotype and partial or complete FSH resistance. ${ }^{5-10,12,13}$
Mutations were mainly identified in individuals from Finland $d^{5,6,8,10}$ and from France. ${ }^{7,9,12}$ To our knowledge, only 10 different inactivating FSHR mutations have been published since 1995 up to now, making this a very rare condition.

Besides mutations with complete FSH receptor inactivation and an early blocked follicular maturation as in prepubertal ovaries, ${ }^{5,6}$ partially inactivating mutations were reported. ${ }^{7,9,19}$ In these patients, the ovaries were of normal size containing follicles developed up to the early antral stages. This observation suggested a limited FSH effect to be sufficient for promoting follicular growth up to the small antral stage. This is in line with Meduri et al, ${ }^{19}$ who hypothesized the absolute requirement of FSH for follicular development starting from the primary stage in humans, in contrast to the initial gonadotrophin-independent follicular growth before the preantralearly antral stages observed in rodents.

Apart from complete and partially inactivating mutations, a modifying effect of SNPs on FSHR function is discussed in the literature..$^{27,28}$ Nakamura et $a l^{13}$ reported on a patient with primary amenorrhea but normal sized ovaries containing some small antral follicles. These follicles reacted to high doses of hMG injection. Molecular analysis revealed only one heterozygote mutation in exon 8 (Val221Gly) and two SNPs in exon 10 (Ala307Thr and Ser680Asn). The authors hypothesized that the different phenotype might be caused by this constellation.

In 2003, Allen et al published a novel inactivating mutation in exon 10 in a female patient with hypergonadotropic ovarian failure and primary amenorrhea. ${ }^{11}$ The mutation was diagnosed as homo- or hemizygous and characterized as complete loss-of-function. Parental analysis could identify the same mutation in the father but not in the mother. A possible uniparental disomy or large deletion was excluded by microsatellite analysis. A translocation was ruled out by normal conventional cytogenetic analysis and whole chromosome painting for chromosome 2. As the chosen microsatellites adjacent to the FSHR locus were 2.1 and $1.5 \mathrm{Mb}$ apart, there was a gap of about $3.6 \mathrm{Mb}$ around the FSHR gene so that a deletion seemed to be possible. The authors suspected either a small deletion on the maternal allele or a gene conversion as two possible explanations but did not prove either hypothesis.

To find out whether there are additional patients with a deletion that might perhaps carry a possible compound heterozygote point mutation, we checked the database for chromosomal imbalances (Decipher) for deletions in this region. At that time (July 2009), the database included three deletion patients. Two patients carry larger deletions, including the FSHR gene $(10.5$ and $1.55 \mathrm{Mb}$ in size, respectively) without any clinical data that would fit in with $\mathrm{FSH}$ resistance. A third patient carries a maternally inherited $120 \mathrm{~kb}$ deletion within the FSHR gene. He shows a congenital malformation/mental retardation syndrome with 36 clinical features (among them brain abnormalities, a congenital heart defect, deafness, craniofacial dysmorphism, macrocephaly, and mental retardation). We suppose that there must be a different, up to now unrecognized reason underlying his phenotype.

The FSHR is a member of the class A G-protein-coupled receptor (GPCR) family (rhodopsin-like), where a proline in TMH6 at the corresponding position of Pro578 is highly conserved. The proline contributes to the formation of a kink in the helix and divides the helix into segments (Figure 3). It is known that such helix-kink distortions are locked by intramolecular side chain interactions between the tightly packed helices. ${ }^{29,30}$ The kink in TMH6 is particularly important for activation and signal transduction in class A G-protein-coupled receptors. This general process is achieved by a 
shift in the spatial arrangement of the transmembrane helices. ${ }^{31}$ In the activation-related scenario, TMH6 undergoes the most important movement where the kink functions as a pivot and finally leads to G-protein coupling and activation. ${ }^{32,33}$ Substitution of the proline is expected to lead to modifications in the signaling properties by causing changes in the helix conformation, intramolecular interactions and thereby the helix arrangement. This is supported by recent findings regarding corresponding prolines in other glycoprotein hormone receptors, the thyrotropin receptor (hTSHR, Pro639), ${ }^{34}$ and the lutropin/choriogonadotropin receptor (rLHCGR, Pro588). ${ }^{35}$ Our performed molecular homology models of the FSHR suggest slight changes in the helix 6 distortion caused by a Pro-His substitution (Figure 3). In consequence, this FSHR variant lost the intrinsic signaling capability determined also by the arrangement of the helical bundle in the basal conformation.

In summary, inactivating FSHR mutations are a very rare cause of primary or early secondary amenorrhea combined with arrested follicular maturation and anovulatory infertility. Depending on the mutation, there is a broad spectrum of clinical phenotypes from complete FSH resistance to incomplete milder forms. Up to now, only point mutations have been found. To our knowledge, our patient presented in this paper is the primary one with a compound heterozygosity of a point mutation and a deletion in the FSHR gene. This deletion of one allele unmasks the mutation of the second allele. The coincidence of two very rare findings - the novel point mutation and the familial unbalanced translocation - leads to an exceptional constellation causing clinical signs consistent with FSH resistance. It should be kept in mind to search for deletions in patients with clinical FSH resistance in whom molecular analysis detected only one heterozygous mutation.

\section{CONFLICT OF INTEREST}

The authors declare no conflict of interest.

\section{ACKNOWLEDGEMENTS}

We are grateful to the patient and her family for their participation in this study. We thank BlueGnome for providing the BAC clones and Hildegard Solka, Lisa Lahrmann and Reinhild Sandhowe-Klaverkamp for excellent technical assistance, Ludger Klein-Hitpaß for performing the SNP array analysis and Dagmar Wieczorek for a critical reading of the article.

\section{WEB RESOURCES}

CNAG V 2.0: http://www.genome.umin.jp/

Decipher: https://decipher.sanger.ac.uk/

http://www.ensembl.org;

http://www.genome.ucsc.edu

http://projects.tcag.ca/variation/

1 Simoni M, Gromoll J, Nieschlag E: The follicle-stimulating hormone receptor: biochemistry, molecular biology, physiology, and pathophysiology. Endocr Rev 1997; 18: 739-773.

2 Themmen APN, Huhtaniemi IT: Mutations of gonadotropins and gonadotropin receptors: elucidating the physiology and pathophysiology of pituitary-gonadal function. Endocr Rev 2000; 21: 551-583. Review.

3 Gromoll J, Ried T, Holtgreve-Grez $\mathrm{H}$ et al: Localization of the human FSH receptor to chromosome 2 p21 using a genomic probe comprising exon 10. J Mol Endocrinol 1994; 12: 265-271.

4 Gromoll J, Pekel E, Nieschlag E: The structure and organization of the human follicle-stimulating hormone receptor (FSHR) gene. Genomics 1996; 35: 308-311.

5 Aittomäki K, Lucena JL, Pakarinen P et al: Mutation in the follicle-stimulating hormone receptor gene causes hereditary hypergonadotropic ovarian failure. Cell 1995; 82: 959-968.

6 Aittomäki K, Herva R, Stenman UH et al: Clinical features of primary ovarian failure caused by a point mutation in the follicle-stimulating hormone receptor gene. J Clin Endocrinol Metabol 1996; 81: 3722-3726.
7 Beau I, Touraine P, Meduri G et al: A novel phenotype related to partial loss of function mutations of the follicle stimulating hormone receptor. J Clin Invest 1998; 102: $1352-1359$.

8 Tapanainen JS, Vaskivuo T, Aittomäki K, Huhtaniemi IT: Inactivating FSH receptor mutations and gonadal dysfunction. Mol Cell Endocrinol 1998; 145: 129-135.

9 Touraine P, Beau I, Gougeon A et al: New natural inactivating mutations of the folliclestimulating hormone receptor: correlations between receptor function and phenotype. Mol Endocrinol 1999; 13: 1844-1854.

10 Doherty E, Pakarinen P, Tiitinen A et al: A Novel mutation in the FSH receptor inhibiting signal transduction and causing primary ovarian failure. J Clin Endocrinol Metab 2002; 87: 1151-1155.

11 Allen LA, Achermann JC, Pakarinen P et al: A novel loss of function mutation in exon 10 of the FSH receptor gene causing hypergonadotrophic hypogonadism: clinical and molecular characteristics. Hum Reprod 2003; 18: 251-256.

12 Meduri G, Touraine P, Beau I et al: Delayed puberty and primary amenorrhea associated with a novel mutation of the human follicle-stimulating hormone receptor: clinical, histological, and molecular studies. J Clin Endocrinol Metab 2003; 88: 3491-3498.

13 Nakamura Y, Maekawa R, Yamagata $Y$ et al: A novel mutation in exon 8 of the follicle-stimulating hormone receptor in a woman with primary amenorrhea. Gynecol Endocrinol 2008; 24: 708-712.

14 Gromoll J, Simoni M, Nieschlag E: An activating mutation of the follicle-stimulating hormone receptor autonomously sustains spermatogenesis in a hypophysectomized man. J Clin Endocrinol Metab 1996; 81: 1367-1370.

15 Smits G, Olatunbosun O, Delbaere A et al: Ovarian hyperstimulation syndrome due to a mutation in the follicle-stimulating hormone receptor. N Engl J Med 2003; 349: 760-766.

16 Vasseur C, Rodien P, Beau I et al: A chorionic gonadotropin-sensitive mutation in the follicle-stimulating hormone receptor as a cause of familial gestational spontaneous ovarian hyperstimulation syndrome. N Engl J Med 2003; 349: 753-759.

17 Montanelli L, Delbaere A, Di Carlo C et al: A mutation in the follicle-stimulating hormone receptor as a cause of familial spontaneous ovarian hyperstimulation syndrome. J Clin Endocrinol Metab 2004; 89: 1255-1258.

18 De Leener A, Montanelli L, Van Durme J et al: Presence and absence of folliclestimulating hormone receptor mutations provide some insights into spontaneous ovarian hyperstimulation syndrome physiopathology. J Clin Endocrinol Metab 2006; 91: 555-562.

19 Meduri G, Bachelot A, Cocca MP et al: Molecular pathology of the FSH receptor: new insights into FSH physiology. Mol Cell Endocrinol 2008; 282: 130-142.

20 Gromoll J, Bröcker M, Derwahl M, Höppner W: Detection of mutation in glycoprotein hormone receptors. Methods 2000; 1: 83-97.

21 Nannya $Y$, Sanada M, Nakazaki $\mathrm{K}$ et al: A robust algorithm for copy number detection using high-density oligonucleotide single nucleotide polymorphism genotyping arrays. Cancer Res 2005; 65: 6071-6079.

22 Kleinau G, Krause G: Thyrotropin and homologous glycoprotein hormone receptors: structural and functional aspects of extracellular signaling mechanisms. Endocr Rev 2009; 30: 133-151.

23 Voigt M, Fusch C, Olbertz D et al: Analysis of the Neonatal Collective in the Federal Republic of Germany. Geburtshilfe und Frauenheilkunde 2006; 66: 956-970.

24 Reinken L, van Oost G: Physical growth of normal German children from birth to 18 years: longitudinal study of height, weight and height velocity. Klin Pädiatr 1992; 204: 129-133.

25 Nellhaus G: Head circumference from birth to eighteen years. Practical composite international and interracial graphs. Pediatrics 1968; 41: 106-114.

26 Rinchik EM, Bultman SJ, Horsthemke B et al: A gene for the mouse pink-eyed dilution locus and for human type II oculocutaneous albinism. Nature 1993; 361: 72-76.

27 Simoni M, Nieschlag E, Gromoll J: Isoforms and single nucleotide polymorphisms of the FSH receptor gene: implications for human reproduction. Hum Reprod Update 2002; 8: 413-421.

28 Greb R, Grieshaber K, Gromoll J et al: A common single nucleotide polymorphism in exon 10 of the human follicle stimulating hormone receptor is a major determinant of length and hormonal dynamics of the menstrual cycle. J Clin Endocrinol Metab 2005; 90: 4866-4872

29 Ceruso MA, Weinstein $\mathrm{H}$ : Structural mimicry of proline kinks: tertiary packing interactions support local structural distortions. J Mol Biol 2002; 318: 1237-1249.

30 Yohannan S, Faham S, Yang D, Whitelegge JP, Bowie JU: The evolution of transmembrane helix kinks and the structural diversity of $\mathrm{G}$ protein-coupled receptors. Proc Natl Acad Sci USA 2004; 101: 959-963.

31 Nygaard R, Frimurer TM, Holst B, Rosenkilde MM, Schwartz TW: Ligand binding and micro-switches in 7TM receptor structures. Trends Pharmacol Sci 2009; 30: 249-259.

32 Altenbach C, Kusnetzow AK, Ernst OP, Hofmann KP, Hubbell WL: High-resolution distance mapping in rhodopsin reveals the pattern of helix movement due to activation. Proc Natl Acad Sci USA 2008; 105: 7439-7444.

33 Scheerer P, Park JH, Hildebrand PW et al: Crystal structure of opsin in its G-proteininteracting conformation. Nature 2008; 455: 497-502.

34 Agretti P, De Marco G, Collecchi P et al: Proper targeting and activity of a nonfunctioning thyroid-stimulating hormone receptor ( $\mathrm{TSHr}$ ) combining an inactivating and activating TSHr mutation in one receptor. Eur J Biochem 2003; 270: 3839-3847.

35 Fernandez LM, Puett D: Identification of amino acid residues in transmembrane helices $\mathrm{VI}$ and $\mathrm{VII}$ of the lutropin/choriogonadotropin receptor involved in signaling. Biochemistry 1996; 35: 3986-3993. 